\title{
Auditory context effects in picture naming investigated with event-related fMRI
}

\author{
Greig I. De Zubicaray and Katie L. McMahon \\ University of Queensland, Brisbane, Queensland, Australia
}

\begin{abstract}
Naming an object entails a number of processing stages, including retrieval of a target lexical concept and encoding of its phonological word form. We investigated these stages using the picture-word interference task in an fMRI experiment. Participants named target pictures in the presence of auditorily presented semantically related, phonologically related, or unrelated distractor words or in isolation. We observed BOLD signal changes in left-hemisphere regions associated with lexical-conceptual and phonological processing, including the midto-posterior lateral temporal cortex. However, these BOLD responses manifested as signal reductions for all distractor conditions relative to naming alone. Compared with unrelated words, phonologically related distractors showed further signal reductions, whereas only the pars orbitalis of the left inferior frontal cortex showed a selective reduction in response in the semantic condition. We interpret these findings as indicating that the word forms of lexical competitors are phonologically encoded and that competition during lexical selection is reduced by phonologically related distractors. Since the extended nature of auditory presentation requires a large portion of a word to be presented before its meaning is accessed, we attribute the BOLD signal reductions observed for semantically related and unrelated words to lateral inhibition mechanisms engaged after target name selection has occurred, as has been proposed in some production models.
\end{abstract}

Naming an object entails a number of processes prior to articulation: The object needs to be identified, its name retrieved from among alternatives in the mental lexicon, and its phonological word form encoded (e.g., Dell, 1986; Harley, 1993; Levelt, Roelofs, \& Meyer, 1999; Rapp \& Goldrick, 2000; Starreveld \& La Heij, 1995). Selection of the appropriate name is generally assumed to occur via a competitive process (e.g., a picture of a horse activates the word horse and also activates the semantically related words cow and $d o g$ ). Speech errors in which an intended word is substituted with a semantically related word support this notion (e.g., Harley \& MacAndrew, 2001). Findings from picture-word interference (PWI) tasks (e.g., Rosinski, Golinkoff, \& Kukish, 1975), in which target pictures are named in the presence of to-be-ignored distractor words, are also interpreted as supporting lexical competition. The empirical observation of semantic interference (SI), the slowing of naming latencies to target objects in the presence of categorically related relative to unrelated distractor words, is considered to be evidence for increased activation of lexical-conceptual competitors during name retrieval (for a review, see Goldrick, 2007; cf. Mahon, Costa, Peterson, Vargas, \& Caramazza, 2007). Another empirical finding is that latencies are shorter when pictures are named in the presence of phonologically related words relative to unrelated words (e.g., presenting the distractor word hose facilitates naming of a picture of a horse relative to the word tree). This facilita- tion effect is viewed as evidence for the activation of overlapping feature-based phonological representations (e.g., Damian \& Bowers, 2009; Schriefers, Meyer, \& Levelt, 1990). Because of word-form overlap, competitive activity is reduced relative to an unrelated word.

Theoretical perspectives of PWI effects are exemplified in connectionist models of language production (e.g., Dell \& O'Seaghdha, 1991; Harley, 1993; Roelofs, 1992). In these models, levels of lexical representation are viewed as patterns of activation, and processing between representational levels is accomplished by spreading activation (see Goldrick, 2007, for an overview). Several issues remain controversial, despite the abundance of behavioral data: The first is whether lexical competitors are phonologically encoded (a process termed phonological coactivation; e.g., Dell, 1986; Harley, 1993), or whether word-form encoding occurs only for a single lexical concept corresponding to the target picture name (e.g., Levelt et al., 1999; cf. Roelofs, 2008b). The second is whether feedback, or interactive activation, occurs between wordform and lexical-conceptual levels (see Goldrick, 2006), phonological coactivation being a prerequisite. The third concerns the possible mechanism(s) for competitor deactivation. Decay-based mechanisms assume that activation returns to a baseline or resting state at some specified period of time after selection of the target name, whereas inhibition-based mechanisms assume that a selected target will then suppress its own activation (e.g., Dell \& 
O'Seaghdha, 1991) and/or the activation of possible competitors, resulting in a temporary state of reduced responsiveness (e.g., Berg \& Schade, 1992; see also Vitkovitch, Rutter, \& Read, 2001).

Neuroimaging and electrophysiological studies of language production have addressed some of these issues. Evidence from these studies indicates that the midportion of the left middle temporal gyrus (MTG) and left posterior MTG/ superior temporal gyrus (STG) mediate lexical-conceptual and word-form processing, respectively (for a review and meta-analysis, see Indefrey \& Levelt, 2004). In terms of specific PWI effects, an fMRI study has demonstrated that SI is associated with increased activation in both of these regions, providing support for phonological coactivation of lexical competitors (e.g., de Zubicaray, Wilson, McMahon, \& Muthiah, 2001; see Mechelli, Josephs, Lambon Ralph, McClelland, \& Price, 2007, for a similar result in the mid and posterior MTG for combinations of written word and picture pairings). Experiments with other naming paradigms that induce SI have likewise revealed signal increases in these regions (e.g., competitor priming; de Zubicaray, McMahon, Eastburn, \& Pringle, 2006). In contrast, phonological facilitation is associated with decreased activation in the left posterior STG, consistent with reduced competition due to word-form overlap (e.g., de Zubicaray, McMahon, Eastburn, \& Wilson, 2002; see Bles \& Jansma, 2008, for a similar result with picture-picture stimuli).

These and other studies have also demonstrated increased activation in the anterior cingulate cortex (ACC), particularly during SI in naming tasks (e.g., de Zubicaray et al., 2006; Hirschfeld, Jansma, Boelte, \& Zwitserlood, 2008; see also Heim, Friederici, Schiller, Rüschemeyer, \& Amunts, 2009). Given that the ACC has been implicated in conflict monitoring across a variety of cognitive tasks (Botvinick, Cohen, \& Carter, 2004), it seems plausible that this activation could represent monitoring of competition among multiple lexical representations during speech production (see de Zubicaray et al., 2001). ERP studies of naming errors have also shown that the error-related negativity elicited by the ACC is larger following distractors that are semantically related to a target picture (e.g., Ganushchak \& Schiller, 2008). Alternatively, the involvement of the ACC in language production might reflect the operation of an executive system that sets and maintains the overall task goal, an operation that is made more or less difficult by the presentation of a semantically or phonologically related distractor word (Roelofs, 2003).

The above interpretations necessarily rely on an assumption that distractor words automatically activate lexical-conceptual and word-form representations. However, in the neuroimaging studies of PWI effects conducted to date, written distractors have generally been employed (e.g., de Zubicaray et al., 2002; de Zubicaray et al., 2001; Hirschfeld et al., 2008). Orthographic and phonological characteristics tend to be closely confounded in many languages, thereby introducing some uncertainty as to the actual derivation of word-form effects elicited by written distractors (Damian \& Bowers, 2009; Damian \& Martin, 1999; Schriefers et al., 1990). In fact, there are ongoing debates about whether visually presented words are processed by independent orthographic or phonologically mediated mechanisms (see Harm \& Seidenberg, 2004) and whether they elicit independent orthographic effects in PWI tasks (see Damian \& Bowers, 2009). Overall, this suggests that the existing data from neuroimaging investigations of PWI tasks using written distractors cannot be interpreted unequivocally.

Several PWI studies have employed auditory distractors, showing behavioral results similar to those in which written distractors were used (e.g., Damian \& Bowers, 2009; Damian \& Martin, 1999; Schriefers et al., 1990). However, presentation of auditory distractors is extended relative to that of written words, occurring over hundreds of milliseconds, complicating the choice of an optimal stimulus onset asynchrony (SOA). For example, phonological facilitation effects have typically not been observed at early SOAs in PWI studies using auditory distractors across English, German, and Dutch (i.e., at $-200 \mathrm{msec}$ and earlier; e.g., Damian \& Martin, 1999; Meyer \& Schriefers, 1991; Starreveld, 2000). The absence of the effect at early SOAs is attributed to the distractor's word-form representation being processed before that of the target (see Starreveld, 2000) and may explain why, in a recent fMRI experiment of PWI, increased activation was observed only in the left supramarginal gyrus when phonological distractors were presented at an SOA of $-200 \mathrm{msec}$ (Abel et al., 2009; this was associated with a "marginally significant" facilitation effect relative to unrelated words). This study likewise failed to observe any differential activity for categorically related relative to unrelated distractors (cf. de Zubicaray et al., 2001; Mechelli et al., 2007). These discrepant results may be due to a number of methodological issues associated with the study design, including the early SOA used, subjective (i.e., manual) determination of response latencies, and use of a conventional continuous fMRI acquisition. Gradient noise is well known to activate temporal lobe regions involved in auditory processing and to interfere with auditory stimulus presentation (see Amaro et al., 2002).

In the present study, we adopted an SOA of $0 \mathrm{msec}$ within a sparse-design fMRI experiment. In addition to having used this SOA with written distractors in our previous fMRI studies, PWI studies with auditory distractors have shown relatively reliable effects of both SI and phonological facilitation around this SOA in English (e.g., Damian \& Bowers, 2009; Damian \& Martin, 1999) and German (e.g., Hantsch, Jescheniak, \& Schriefers, 2005; Jescheniak \& Schriefers, 1998; Schriefers et al., 1990). We expected to replicate the results of our earlier fMRI studies of PWI effects with semantically and phonologically related distractors (described above). Unlike our earlier investigations, in this study, we included a naming-only condition in order to assist with the localization of distractor effects. Since the presentation of distractor words produces an interference effect when naming a target picture, we expected the distractor conditions to elicit increased activation in the mid-to-posterior temporal MTG and STG and in the ACC relative to naming in isolation. 


\section{METHOD}

\section{Participants}

A total of 17 healthy participants (14 male), with a mean age of 26.12 years $(S D=3.97)$, performed the experiments. They were recruited from undergraduate and postgraduate students and staff of the University of Queensland. All were right-handed native English speakers, with no history of neurological or psychiatric disorder or substance dependence. They had no known hearing deficits and had normal or corrected-to-normal vision. They were reimbursed for participating.

\section{Materials}

Twenty black-and-white line drawings from a range of semantic categories were selected from the Snodgrass and Vanderwart (1980) set of pictures to serve as targets. For each picture, a categorically related word was selected that served as the semantically related distractor; a word that shared the initial consonant-vowel segment was selected as the phonologically related distractor; and a word with no discernible relationship to the picture was selected as an unrelated distractor. Distractor words were selected from a subset of words used by Damian and Martin (1999; see their Supplementary Material Section). The lexical properties of these words are shown in Table 1. The distractor words were matched according to mean frequencies of occurrence, numbers of letters, syllables, phonemes, orthographic and phonological neighbors, and mean durations (in milliseconds; all $F \mathrm{~s}<1$ ).

\section{Apparatus}

To construct the distractors, the selected words were spoken at the rate of one every $3 \mathrm{sec}$ by a female native English speaker into a Røde NT3 condenser microphone in a soundproof room and recorded as a continuous digital audio file (sampling rate $44 \mathrm{kHz}$ ) using a Roland VS880 workstation and postequalized. The resulting audio file was then segmented into separate digitized words using CoolEdit software (Syntrillium Software). A laptop PC running Microsoft Visual Basic and ExacTicks (Ryle Design) software was used to deliver the picture and word stimuli and to record vocal responses on digital audio files (sampling rate $11 \mathrm{kHz}$ ). Line drawings were presented in black on a luminous white background, enlarged and back-projected onto a screen that the participants viewed through a mirror mounted on the head coil using a BenQ SL705X projector. The pictures subtended approximately $10^{\circ}$ of visual arc when each participant was positioned for imaging. Distractor words were presented through magnetic resonance (MR) compatible earpieces consisting of GELseal eartips with the audio volume adjusted for each participant prior to imaging. An additional $30-\mathrm{dB}$ attenuating headset was used to reduce gradient noise. Naming responses were recorded using a custom positioned MR-compatible microphone attached to the head coil. Naming latencies were determined with voice-key software. The filtered audio files were consulted for scoring responses.

\section{Procedure}

The procedure closely followed that of behavioral studies of PWI effects. Participants were first familiarized with the set of experimental pictures by viewing each one on a laptop computer screen in random order with the appropriate label printed below. The size of the pictures including the background was approximately $10 \mathrm{~cm}$ wide $\times 10 \mathrm{~cm}$ high. This occurred over two consecutive practice blocks in which the participants were instructed to name the pictures as fast and as accurately as possible. Erroneous naming responses were corrected. In a final practice block, they viewed the pictures without the labels printed below and were instructed to name the pictures as per the previous instructions.

The experimental session, consisting of two separate blocks of 40 trials each, was then conducted (20 target pictures presented in four conditions: no distractor, semantically related, phonologically related, and unrelated distractor words). A short break was permitted between the two blocks while a structural image was acquired (see the Image Acquisition section below). A pseudorandom order for trial presentation was adopted across participants, such that two presentations of the same picture were always interceded by at least 5 different pictures in order to diminish possible item repetition effects. Participants were instructed to name the pictures as quickly and accurately as possible while ignoring the distractor. In the event of a picture-naming error, they were instructed not to correct their response and to wait for the next trial. They were also instructed not to speak or move during image acquisition (as indicated by the relatively loud gradient noise). The presentation of a trial involved the following sequence: A fixation point $(+)$ was shown for $50 \mathrm{msec}$ in the center of a screen, followed by the auditory presentation of the distractor word and the picture displayed in the middle of the screen at an SOA of $0 \mathrm{msec}$. The picture remained on the screen for $750 \mathrm{msec}$ (see de Zubicaray et al., 2001, for a diagram of the experimental sequence). Following the completion of the experimental session, participants were questioned regarding the clarity of their perception of the spoken distractors in order to ensure the quality of the data acquired.

Image acquisition. Participants were imaged with a Bruker Medspec system operating at $4 \mathrm{~T}$ using a transverse electromagnetic head coil for radiofrequency transmission and reception (Vaughn et al., 2002). A gradient echo planar imaging (EPI) sequence optimized for both image quality and noise reduction (McMahon, Pringle, Eastburn, \& Maillet, 2004) was used to acquire T2*-weighted images depicting BOLD contrast $(64 \times 64$ matrix; $3.6 \times 3.6-\mathrm{mm}$ voxels). In a single session, 82 image volumes of 36 axial $3.5-\mathrm{mm}$ slices (0.1-mm gap) were acquired (effective repetition time, $15 \mathrm{sec}$; echo time, $30 \mathrm{msec}$; flip angle, $90^{\circ}$ ). The first two volumes were discarded. Behavioral trials were interleaved with image acquisition using sparse temporal sampling to capture the estimated peak BOLD signal response to task-related neural activity (Eden, Joseph, Brown, Brown, \& Zeffiro, 1999; Elliott, Bowtell, \& Morris, 1999). For each trial, no field gradients were applied for a 4 -sec period of relative silence, allowing for stimulus presentation and the participant's overt verbal response, then immediately applied for image acquisition. A single image volume was then acquired within $3 \mathrm{sec}$, approximately coincident with the trial's estimated peak BOLD response. No field gradients were applied for an additional 8-sec period to allow the BOLD response to the gradient noise to return to baseline (for a schematic of the imaging protocol, see Figure 1 in de Zubicaray et al., 2001). Total imaging time was approximately $20 \mathrm{~min}$. Head movement was limited by foam padding within the head coil. A point-spread function mapping sequence was acquired prior to the EPI time-series acquisitions to correct geometric distortions (Zait-

Table 1

Mean Lexical Properties of Distractor Stimuli

\begin{tabular}{|c|c|c|c|c|c|c|c|}
\hline & \multicolumn{3}{|c|}{ Word Length } & \multirow{2}{*}{$\begin{array}{c}\text { CELEX } \\
\text { Frequency }\end{array}$} & \multicolumn{2}{|c|}{ Neighbors ${ }^{b}$} & \multirow{2}{*}{$\begin{array}{c}\text { Duration } \\
(\mathrm{msec})\end{array}$} \\
\hline & Letters & Syllables & Phonemes & & Phonological & Orthographic & \\
\hline Unrelated & 4.5 & 1.35 & 3.75 & 20.68 & 14.05 & 6.85 & 555.20 \\
\hline Semantically related & 4.55 & 1.25 & 3.55 & 20.96 & 12.9 & 5.55 & 567.95 \\
\hline Phonologically related & 4.5 & 1.25 & 3.8 & 28.61 & 16.15 & 7.65 & 563.55 \\
\hline
\end{tabular}

aFrequency per million. Source: Baayen, Piepenbrock, and Gulikers (1995). bSource: Davis (2005). 
sev, Hennig, \& Speck, 2003). Finally, a 3D T1-weighted image was acquired using a magnetization prepared rapid acquisition gradient echo sequence ( $256^{3}$ matrix; $0.9-\mathrm{mm}^{3}$ voxels).

Image analysis. The first two volumes from the fMRI time series were discarded, and the remaining images were motion corrected using INRIAlign (Freire, Roche, \& Mangin, 2002). A mean image was generated from the realigned series; the series was regrouped, with images from each distractor condition comprising a single epoch; and trials meeting exclusion criteria were removed (see the Behavioral Data section below). The resulting images were analyzed using statistical parametric mapping software (SPM5; Wellcome Department of Imaging Neuroscience, Queen Square, London). The realigned image volumes from each participant were spatially normalized to MNI atlas space using the linear and nonlinear transformations from their coregistered T1-weighted image. The resulting images were resampled to $3-\mathrm{mm}^{3}$ voxels and smoothed with a 9-mm FWHM isotropic Gaussian kernel.

We conducted a two-stage mixed effects model statistical analysis. Epoch types corresponding to the distractor conditions were modeled as effects of interest, with delta functions representing each epoch onset, and convolved with a basis function consisting of a single finite impulse response encompassing the epoch length. Highand low-pass filtering were not applied because of the reordering of the time series data and use of long TRs (see de Zubicaray et al., 2006). Linear contrasts were applied to each participant's parameter estimates at the fixed effects level and then entered in a group-level repeated measures ANOVA in which covariance components were estimated using a restricted maximum likelihood procedure to correct for nonsphericity (Friston et al., 2002).

A priori regions of interest (ROIs) were defined for small volume corrections using labeled maximum likelihood gray matter maps from a 3-D probabilistic atlas (Shattuck et al., 2008). These included the following left-hemisphere regions: middle and superior temporal gyri (combined), and inferior frontal gyrus. ACC (defined as $y>61$ ) ROIs were defined for both hemispheres. In addition to a wholebrain alpha threshold of $p<.05$ corrected for multiple comparisons using the false discovery rate (FDR) method (Genovese, Lazar, \& Nichols, 2002), we report only those effects surviving an FDR correction at $p<.05$ within the a priori defined ROIs. In order to compare conditions, percentage BOLD signal responses were extracted from regions showing a significant main effect using MarsBaR software (v 0.41; marsbar.sourceforge.net).

\section{RESULTS}

\section{Behavioral Data}

Incorrect or omitted responses $(2.1 \%)$ were excluded from the response latency analysis. Following previous auditory PWI studies (e.g., Damian \& Bowers, 2009; Starreveld, 2000), latencies faster than $250 \mathrm{msec}$ or slower than $1,500 \mathrm{msec}$ were considered outliers and likewise excluded (5.8\%). Table 2 presents the mean latencies and error proportions according to distractor condition (unrelated, semantically related, phonologically related, and control/no distractor). An ANOVA was conducted on the response latency means with distractor type as a withinparticipants variable. We found a main effect of distractor type $\left[F(1,16)=27.22, M S_{\mathrm{e}}=28,947, p<.001\right]$. Overall, paired $t$ tests revealed longer naming latencies for all distractor types relative to the control (no-distractor) condition [unrelated $t(16)=6.25, p<.001$; semantically related, $t(16)=6.91, p<.001$; phonologically related, $t(16)=2.86, p<.05]$. Longer naming latencies were observed for the semantically related distractors than for the other distractor types [unrelated, $t(16)=2.44, p<.05$;
Table 2

Mean Response Latencies (in Milliseconds) and Mean Percentages of Error According to Distractor Condition,With Standard Deviations

\begin{tabular}{lcccc}
\hline \multicolumn{1}{c}{ Condition } & $\begin{array}{c}\text { Response } \\
\text { Latency }\end{array}$ & SD & $\begin{array}{c}\text { Error } \\
\text { Percentage }\end{array}$ & $S D$ \\
\hline Unrelated & 914 & 300 & 3.5 & 5.5 \\
Semantically related & 935 & 290 & 2.6 & 4.7 \\
Phonologically related & 879 & 289 & 1.2 & 2.8 \\
Control & 841 & 278 & 1.2 & 3.8 \\
\hline
\end{tabular}

phonologically related, $t(16)=5.16, p<.001]$, whereas shorter naming latencies were observed for phonologically related distractors than for the other distractor types [unrelated, $t(16)=-4.45, p<.001$ ]. A similar ANOVA was conducted on the error percentages, showing no significant effect of distractor condition $[F(1,16)=2.15$, $\left.M S_{\mathrm{e}}=22.92\right]$.

\section{fMRI Data}

ROI analyses. Two of the four ROIs interrogated showed significant main effects of distractor type at $p<$ .05 (FDR corrected): left middle and superior temporal gyri (combined) and inferior frontal gyrus. However, the peaks in the temporal lobe were part of a single contiguous cluster encompassing the mid-through-posterior portions of the middle and superior temporal gyri (see Figure 1). ${ }^{1}$ Within the left inferior frontal gyrus ROI, two peaks were identified corresponding to the pars triangularis and pars orbitalis (BAs 45 and 47, respectively; Figure 1 and Table 3). No statistically significant effects were observed in either of the ACC ROIs.

Paired $t$ tests were conducted on the mean percentage BOLD signal differences (distractor minus no distractor) extracted from the clusters showing a significant main effect of distractor type. Within the mid-posterior left temporal cortex, phonologically related distractors showed a significantly decreased response relative to unrelated distractors $\left[t_{16}=-4.33, p<.001\right]$ and a nonsignificant trend in the same direction relative to the semantically related distractors $[t(16)=-1.86, p=.08]$. Responses to semantically related distractors did not differ significantly from

Table 3

Peak Maxima for the Main Effect of Distractor Type

\begin{tabular}{lcc}
\hline \multicolumn{1}{c}{ Cerebral Region } & Coordinates $(x, y, z)$ & $z$-Score \\
\hline Left middle temporal gyrus & $\mathbf{- 5 4 , - 1 8 , 0}$ & $\mathbf{5 . 7 8}$ \\
Left superior temporal gyrus & $\mathbf{- 6 0 , - 3 0 , 3}$ & $\mathbf{5 . 3 4}$ \\
& $-63,-48,15$ & 3.4 \\
& $63,-27,6$ & 5.35 \\
Right superior temporal gyrus & $-\mathbf{3 6},-\mathbf{2 7 , 1 2}$ & $\mathbf{3 . 4 2}$ \\
Left Heschl's gyrus & $\mathbf{- 4 8 , 2 1 , \mathbf { 1 8 }}$ & $\mathbf{3 . 8 7}$ \\
Left inferior frontal gyrus & & \\
$\quad$ (pars triangularis) & $\mathbf{- 5 1 , 2 4 , - 9}$ & $\mathbf{3 . 1 4}$ \\
$\quad$ Left inferior frontal gyrus & $-12,-69,24$ & 4.08 \\
$\quad$ (pars orbitalis) & $-30,-93,3$ & 3.53 \\
\hline Left cuneus &
\end{tabular}

Note-Peak coordinates thresholded at $p<.05$ (false discovery rate corrected across the whole brain). Peak coordinates within regions of interest that exceeded a threshold of $p<.05$ (false discovery rate small volume corrected) are shown in bold. 

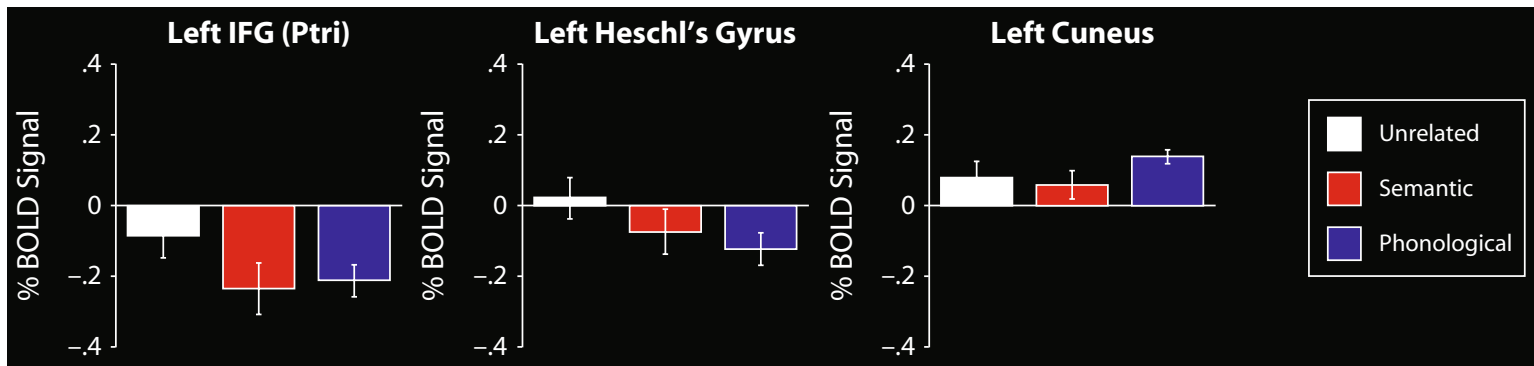

Left Hemisphere (Lateral)

Left Hemisphere (Medial)

Right Hemisphere (Lateral)
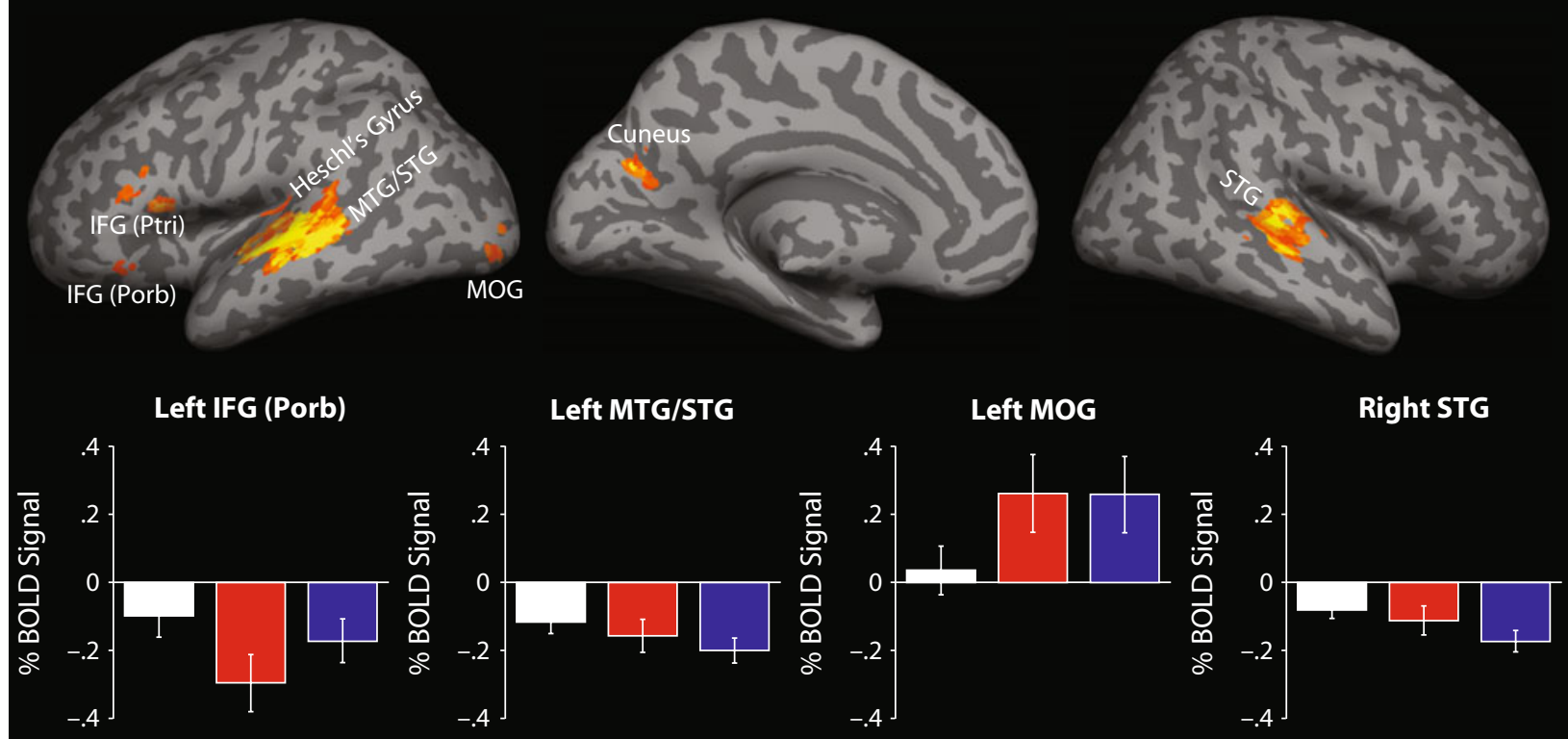

Figure 1. Middle panel: Cerebral regions showing a significant main effect of distractor type in the whole-brain analysis (thresholded at $p<.001$ and $p>5$ contiguous voxels per cluster for visualization purposes), displayed on the inflated cortical surface of an individual brain. Top and bottom panels: BOLD signal difference plots (distractor minus no distractor) extracted from clusters showing a significant main effect of distractor type. IFG, inferior frontal gyrus; Porb, pars orbitalis; Ptri, pars triangularis; MTG, middle temporal gyrus; STG, superior temporal gyrus; MOG, middle occipital gyrus.

those to unrelated distractors $[t(16)=-1.55, p=.14]$. In the pars triangularis of the inferior frontal gyrus, related distractors showed significantly reduced responses relative to unrelated ones [semantically related, $t(16)=-2.5$, $p<.05$; phonologically related, $t(16)=3.97, p<.001]$, although they did not differ themselves $[t(16)=-.42$, $p=.68]$. Within the pars orbitalis of the left IFG, semantically related distractors showed a significantly decreased response relative to those of the other two distractor types [unrelated, $t(16)=-3.41, p<.005$; phonologically related $t(16)=-2.52, p<.05]$, with the latter two distractors not differing significantly $[t(16)=1.67, p=.11]$.

Whole-brain exploratory analyses. This analysis revealed a predominantly left-hemisphere network of regions showing a main effect of distractor type, including most of the regions identified in the ROI analyses (Table 3). This network incorporated additional regions primarily in the auditory and visual cortices: Heschl's gyrus (BA 41), the middle occipital gyrus, and the calcarine sulcus/cuneus (BAs 17/18). In the right hemisphere, only the posterior STG showed a main effect of distractor type.
The cluster within Heschl's gyrus showed a significantly decreased response for related relative to unrelated distractors [semantically related, $t(16)=-3.33, p<.005$; phonologically related, $t(16)=-3.91, p<.001]$, although there was no difference between related distractor types $[t(16)=-1.59, p=.13]$. In the right hemisphere, the posterior STG showed a significantly decreased response for phonologically related compared with unrelated distractors $[t(16)=-3.83, p<.001]$, and a trend in the same direction relative to semantically related distractors $[t(16)=-1.93, p<.08]$. Responses to semantically related distractors did not differ significantly from those to the unrelated distractors $[t(16)=-1.2, p=.25]$.

The two remaining clusters in the left primary visual cortex demonstrated significant differences in BOLD signal responses between conditions that were in the opposite direction from those observed elsewhere: The middle occipital gyrus showed a significant effect of relatedness, demonstrating signal increases for related relative to unrelated distractors [semantically related, $t(16)=2.87, p<$ .05 ; for phonologically related, $t(16)=3.5, p<.005$ ], and 
no difference between related distractor types $[t(16)=$ $.03, p=.98]$. Finally, in the cuneus/calcarine sulcus, the related distractors differed significantly, with phonologically related items showing increased signal relative to semantically related distractors $[t(16)=-2.5, p<.05]$, although neither related distractor type differed significantly from unrelated distractors [semantically related, $t(16)=-.69, p=.49$; phonologically related, $t(16)=$ $1.45, p=.16]$.

\section{DISCUSSION}

Relatively few neuroimaging studies have examined PWI effects. The present study is the first, to our knowledge, in which auditory distractors were employed within an fMRI experiment of PWI and in which a naming-only condition was included to investigate distractor competition effects (cf. Abel et al., 2009). Naming latencies were longer when distractor words were presented than when they were not, and latencies in the different distractor conditions conformed to patterns reported previously: Semantically related distractors produced interference, and phonologically related distractors facilitated naming relative to unrelated distractors. Overall, distractor effects were associated with robust BOLD signal responses in the left-mid-to-posterior MTG/STG, and phonologically related distractors showed an expected decrease in BOLD signal relative to unrelated words. However, the direction of the responses for semantically related and unrelated words differed from that of previous studies, manifesting as decreases in BOLD signal within the MTG/STG, although not to the same extent observed for word-form-related distractors.

The chief finding of the present study is that auditory context effects in PWI are associated with differential activity throughout the mid-to-posterior portions of the left MTG and STG, cortical regions associated with lexicalconceptual selection and word-form encoding, respectively (Indefrey \& Levelt, 2004). This result supports the view that the processing of lexical competitors and their corresponding word forms overlaps during language production. It is therefore consistent with recent empirical work indicating that alternative nonselected names are phonologically activated, even if they are inappropriate in context (e.g., Jescheniak, Hantsch, \& Schriefers, 2005). Phonological coactivation is a key assumption of cascade and interactive models of language production (e.g., Dell, 1986; Harley, 1993; Peterson \& Savoy, 1998; Rapp \& Goldrick, 2000), in contrast to serial models (e.g., Levelt et al., 1999; Roelofs, 1992). Recently, Roelofs (2008b) yielded the serial model position to permit limited spreading of activation between lexical concepts and word forms.

However, contrary to previous findings with written distractors (e.g., de Zubicaray et al., 2002; de Zubicaray et al., 2001), auditory distractor effects manifested as signal reductions in the majority of cortical regions showing BOLD responses. This was the case across unrelated and semantically and phonologically related conditions relative to naming alone. Although unexpected, there may be a number of reasons that this occurred. First, many fMRI studies have demonstrated that selective attention modulates BOLD responses in regions associated with processing of target stimuli. Specifically, in crossmodal auditory-visual tasks, ignored stimuli are associated with attenuated activation in sensory cortical regions engaged when the stimuli are attended (e.g., Baier, Kleinschmidt, \& Müller, 2006; Laurienti et al., 2002; Weissman, Warner, $\&$ Woldorff, 2004). In addition, when attending to visual targets, the presentation of auditory distractors enhances BOLD responses in the visual cortex, perhaps because of the operation of a mechanism that amplifies attention to goal-relevant features in the presence of distraction (Baier et al., 2006; Weissman et al., 2004). These responses are also modulated by the relevance of the distracting stimuli (Baier et al., 2006; Weissman et al., 2004). This might explain the signal increases we observed solely in the visual cortex in the related conditions in the present study, as well as the parallel signal decreases in the auditory cortex. Alternatively, the signal increases in the visual cortex might be considered consistent with some recent evidence from a study that employed a psychological refractory period paradigm indicating that the locus of the SI effect in PWI might be at a perceptual encoding stage of processing (Dell'Acqua, Job, Peressotti, \& Pascali, 2007). Because the latter account is based on data using visual distractors, in which pictorial and orthographic information interact early in processing, we do not consider it a likely explanation for the present results with auditory distractors, as we elaborate below. Irrespective of these issues, neither selective attention nor perceptual encoding mechanisms appear sufficient to explain the patterns of BOLD responses observed among the different distractor conditions elsewhere in the association cortex.

The relatively reduced activation associated with phonologically related distractors in the left mid-posterior temporal cortex (and in a homologous region in the right hemisphere) relative to unrelated words might therefore be attributed to reduced processing requirements because of overlapping feature-based phonological representations (Damian \& Bowers, 2009; see Meyer \& Damian, 2007, for a similar interpretation of phonological facilitation with picture-picture stimuli). Because of feature overlap, there is less competitive activity relative to unrelated and semantically related words (Goldrick, 2007). This result, observed in the absence of a confounding influence of orthography, corroborates and extends the findings of earlier fMRI studies employing visual picture-word and picturepicture stimuli (e.g., Bles \& Jansma, 2008; de Zubicaray et al., 2002; cf. Abel et al., 2009). However, an explanation for the signal reductions observed with unrelated and semantically related words is less straightforward, given prior results with written distractors and the interpretations offered in the context of language production models (e.g., de Zubicaray et al., 2001).

A number of authors have noted that the processing of auditory distractors is not coincident with the SOA of presentation and depends on a range of factors, making the interpretation of PWI effects problematic with these stimuli (e.g., Damian \& Martin, 1999; Starreveld, 2000). This is because auditory presentation of distractors takes several hundreds of milliseconds. Most of this time elapses 
before the word is recognized and its meaning extracted. By contrast, the matching initial word-form information of phonologically related distractors is processed earlier, assisting selection. ${ }^{2}$ However, if phonologically related distractors are presented too early, word perception processes predominate and the facilitation effect is typically not observed (e.g., at an SOA of $-200 \mathrm{msec}$; Abel et al., 2009; see Starreveld, 2000, for a discussion). As a consequence of the protracted auditory presentation, in the unrelated and semantically related conditions, the target lexical concept may be selected, and the concept level will begin to return to a stable state while the distractor word is still being processed (Starreveld, 2000; see also Berg $\&$ Schade, 1992). In principle, this could also explain the absence of distractor-related activity in the ACC, a finding that differs from previous work with simultaneously presented written and picture distractors and was contrary to what was expected (e.g., de Zubicaray et al., 2002; de Zubicaray, 2001; Heim et al., 2008; Hirschfeld et al., 2008). Because lexical processing of the target is largely resolved by the time the distractor's lexical concept is accessed, demands on monitoring are reduced. Of note, Abel et al. (2009) likewise failed to observe ACC activity across their distractor conditions, although this result was probably due to the use of an early SOA that allowed distractor processing to precede that of the target.

The timing of SOAs is equally important for the BOLD activity elicited with visual distractors, as the results of a recent fMRI study by Spalek and Thompson-Schill (2008) show. These authors used a late SOA (distractor words were presented $550 \mathrm{msec}$ after picture onset). Because they were unable to obtain voice onset latencies in the scanner, they conducted a separate behavioral experiment in which they failed to observe an SI effect with this SOA (see their Table 1; p. 223), consistent with the literature that shows that the effect is restricted to a small range of SOAs around $0 \mathrm{msec}$ (see Damian \& Martin, 1999). Of note, Spalek and Thompson-Schill did not observe any BOLD signal increases in left middle or superior temporal cortex, or the ACC, suggesting that the distractor word was presented too late to induce lexical competition, unlike earlier studies that used an SOA of 0 msec (e.g., de Zubicaray et al., 2001).

If, in the present study, the target concept was selected in the unrelated and semantically related distractor conditions because the processing of auditory stimuli is extended over time, the BOLD signal reductions observed in the temporal cortex for these conditions need to be explained by a postselection mechanism. A decay-based mechanism entails a return of activation to a baseline/ resting state; it does not predict a signal reduction (e.g., Starreveld, 2000). This is also the case for self-inhibition, in which a selected concept resets its activation to a baseline level (e.g., Dell \& O'Seaghdha, 1991). Therefore, a production model that also involves lateral inhibition appears the most viable (e.g., Berg \& Schade, 1992; Harley, 1993; see Dell \& O'Seaghdha, 1994, and Goldrick, 2007). The strength of lateral inhibition is determined by the number of potential connections between competitors. It develops between lexical neighbors in proportion to their relatedness (see Dell \& O'Seaghdha, 1994; Harley, 1993), resulting in stronger inhibition being applied in the semantically related distractor condition relative to the unrelated condition. Since the purpose of lateral inhibition is to suppress mismatching information at the lexical level in order to permit the target to dominate subsequent processing (see Dell \& O'Seaghdha, 1994; Goldrick, 2007), these connections also operate in the unrelated and phonologically related distractor conditions. In the latter, inhibition would likely act to further reduce activity as soon as the end word-form information began to mismatch that of the target representation. Lateral inhibition is also considered capable of producing a brief state of suppressed responsiveness in the production system after selection has occurred (e.g., Vitkovitch et al., 2001). Thus, this account has the distinct advantage of proposing a common mechanism contributing to the BOLD signal reductions observed across the experimental conditions. It seems reasonable to assume that suppressed/reduced processing activity would manifest as reduced BOLD responsiveness in the context of an fMRI experiment.

Before proceeding with this account, it is worth considering an alternative scenario that might be invoked to explain the present pattern of results. For example, a number of authors consider semantic context effects in naming tasks to reflect contributions from both conceptual facilitation and lexical competition mechanisms, with the latter offsetting the former in the case of SI (e.g., Abdel Rahman \& Melinger, 2009; Damian \& Als, 2005; Kuipers, La Heij, \& Costa, 2006; Navarrete \& Costa, 2005). Conceptual facilitation is usually associated with semantic priming in picture-naming latencies (e.g., Abdel Rahman \& Melinger, 2009; Damian \& Als, 2005; Kuipers et al., 2006). Mahon et al. (2007) have recently proposed a bespoke postlexical selection account for SI in PWI that offsets the conceptual facilitation effect without the involvement of lexical competition. Although it is possible that the BOLD signal reductions in the semantically related condition might represent conceptual facilitation followed by a postlexical response selection mechanism, as Mahon et al. proposed, we consider it unlikely. By linking their postlexical selection mechanism to those proposed in models of Stroop performance, Mahon et al.'s account necessarily predicted that an increase in BOLD signal would be observed. Meta-analyses of results from Stroop neuroimaging studies have identified reliable activation increases in the left IFG, insula, and ACC (e.g., Laird et al., 2005). No signal increases were observed in these regions in the present study.

In our earlier picture-naming fMRI studies, we proposed that central mechanisms can and do intervene during language production (de Zubicaray, 2006; see also Roelofs, 2008a, for a similar proposal). Informed by an intraoperative electrical stimulation study that elicited semantic substitution errors during picture naming (Duffau et al., 2005), we recently proposed that the pars orbitalis of the IFG might have a role in central or top-down modulation of the language production system, biasing lexical selection to meet the current goal of speech (de Zubicaray et al., 2006). ${ }^{3}$ In the present study, this region showed a 
selective reduction in BOLD response in the semantically related distractor condition. Given that this was the only cortical region to show a selective response to semantically related distractors, it is tempting to conclude that the relatively longer naming latencies might therefore reflect the operation of a top-down mechanism mediated by the pars orbitalis, perhaps of an inhibitory nature.

Within the left IFG, the pars triangularis (BA44/45) showed relatively reduced responses for related relative to unrelated distractors. Kan and Thompson-Schill (2004) presented evidence indicating that activity in this region during picture naming is mediated by the degree of selection required among alternative lexical representations. An explanation along these lines could attribute the reduced signal for phonologically related distractors to reduced selection demands because of feature overlap. Another account is needed for the semantically related condition. One possibility is that the reduced signal observed for this distractor condition might reflect diminished selection demands due to lateral inhibition of competing representations (see Vitkovitch et al., 2001). ${ }^{4}$

\section{SUMMARY AND CONCLUSIONS}

At the outset, our intention was to replicate the results of our previous PWI studies in the absence of a confounding effect of orthography. Because of the extended presentation of auditory distractors, the interpretation of PWI effects was less straightforward than that for written distractors, despite the use of an identical SOA. This was also the case for a recent fMRI study that used an early SOA (Abel et al., 2009). Although we replicated previous findings associated with phonological facilitation, we observed BOLD signal reductions where increases had been observed with written distractors for unrelated and semantically related conditions. If, in these latter conditions, the protracted nature of auditory distractors results in target name selection before the distractor's lexical concept is accessed, it follows that the SI effect and the BOLD signal reductions observed need to be explained by postselection mechanisms occurring at the lexical level. Thus, we consider that the BOLD signal reductions in temporal cortical regions associated with lexical conceptual and word-form processing can only be accommodated by language production models incorporating lateral inhibition. In addition, we consider it likely that central mechanisms in anterior cortex intervene in the language production system in order to select words that meet the current goal of speech.

In terms of future work, it might be the case that auditory distractors are less than suitable stimuli for fMRI experiments, since the onsets and offsets of perception and production effects with these stimuli are not certainunlike those of visual distractors (Starreveld, 2000) - and are unlikely to be resolved successfully with the relatively slow temporal resolution of the BOLD response, even with the use of a range of SOAs across conditions (typically in steps of $100 \mathrm{msec}$ in behavioral studies). In addition, the time needed for such an experiment would be well beyond the scope of most fMRI investigations. Future neuroimaging investigators of PWI effects may therefore wish to employ electrophysiological techniques such as MEG or EEG, which have superior temporal resolutions.

Finally, the present study employed a naming-only control condition in order to reliably identify distractor competition effects in PWI. The experimental design therefore differs from that of prior neuroimaging studies that used visually presented distractors contrasted with lexical control conditions (e.g., a row of Xs; de Zubicaray et al., 2001; Hirschfeld et al., 2008). Although this manipulation might have been a factor contributing to the different pattern of results that we observed, we are not aware of any accounts of PWI that hypothesize different patterns of activation due to the inclusion of different control conditions, nor are we aware of any evidence from the behavioral literature indicating that including a naming-only control condition alters the typical pattern of results. In order to address this issue, future researchers performing neuroimaging studies of PWI might consider examining visual distractors with a similar control condition.

\section{AUTHOR NOTE}

We thank Matthew Eastburn and Martina Bryant for their assistance in acquiring the data. We are grateful to three anonymous reviewers for their helpful feedback on a previous version of this article. Correspondence concerning this article should be addressed to G. I. de Zubicaray, Centre for Magnetic Resonance, University of Queensland, QLD, 4072 Australia (e-mail: greig.dezubicaray@uq.edu.au).

\section{REFERENCES}

Abdel Rahman, R., \& Melinger, A. (2009). Semantic context effects in language production: A swinging lexical network proposal and a review. Language \& Cognitive Processes, 24, 713-734. doi:10.1080/01690960802597250

Abel, S., Dressel, K., Bitzer, R., Kümmerer, D., Mader, I., Weiller, C., \& Huber, W. (2009). The separation of processing stages in a lexical interference fMRI-paradigm. NeuroImage, 44, 1113-1124.

Amaro, E., Jr., Williams, S. C., Shergill, S. S., Fu, C. H., MaCSweeney, M., Picchioni, M., ET AL. (2002). Acoustic noise and functional magnetic resonance imaging: Current strategies and future prospects. Journal of Magnetic Resonance Imaging, 16, 497-510.

BaAyen, R. H., Piepenbrock, R., \& Gulikers, L. (1995). The CELEX lexical database [CD-ROM]. Philadelphia: Linguistic Data Consortium, University of Pennsylvania.

Baier, B., Kleinschmidt, A., \& Müller, N. G. (2006). Cross-modal processing in early visual and auditory cortices depends on expected statistical relationship of multisensory information. Journal of Neuroscience, 26, 12260-12265. doi:10.1523/JNEUROSCI.1457-06.2006

Berg, T., \& Schade, U. (1992). The role of inhibition in a spreading activation model of language production, Part 1: The psycholinguistic perspective. Journal of Psycholinguistic Research, 22, 405-434.

Bles, M., \& Jansma, B. M. (2008). Phonological processing of ignored distractor pictures: An fMRI investigation. BMC Neuroscience, 9, 20.

Botvinick, M., Cohen, J. D., \& CARTER, C. S. (2004). Conflict monitoring and anterior cingulate cortex: An update. Trends in Cognitive Sciences, 8, 539-546.

Damian, M. F., \& Als, L. C. (2005). Long-lasting semantic context effects in the spoken production of object names. Journal of Experimental Psychology: Learning, Memory, \& Cognition, 31, 1372-1384.

Damian, M. F., \& Bowers, J. S. (2009). Assessing the role of orthography in speech perception and production: Evidence from pictureword interference tasks. European Journal of Cognitive Psychology, 21, 581-598. doi:10.1080/09541440801896007

Damian, M. F., \& Martin, R. C. (1999). Semantic and phonological 
codes interact in single word production. Journal of Experimental Psychology: Learning, Memory, \& Cognition, 25, 345-361.

DAvis, C. J. (2005). N-Watch: A program for deriving neighborhood size and other psycholinguistic statistics. Behavior Research Methods, 37, 65-70.

DeLL, G. S. (1986). A spreading activation theory of retrieval in sentence production. Psychological Review, 93, 283-321.

Dell, G. S., \& O'Seaghdha, P. G. (1991). Mediated and convergent lexical priming in language production: A comment on Levelt et al. (1991). Psychological Review, 98, 604-614.

Dell, G. S., \& O'Seaghdha, P. G. (1994). Inhibition in interactive activation models of linguistic selection and sequencing. In D. Dagenbach \& T. H. Carr (Eds.), Inhibitory processes in attention, memory, \& language (pp. 409-451). San Diego: Academic Press.

Dell'Acqua, R., Job, R., Peressotti, F., \& Pascali, A. (2007). The picture-word interference effect is not a Stroop effect. Psychonomic Bulletin \& Review, 14, 717-722.

DE Zubicaray, G. I. (2006). Cognitive neuroimaging: Cognitive science out of the armchair. Brain \& Cognition, 60, 272-281.

de Zubicaray, G. I., McMahon, K. L., Eastburn, M. M., \& PrinGLE, A. (2006). Top-down influences on lexical selection during spoken word production: A 4T fMRI investigation of refractory effects in picture naming. Human Brain Mapping, 27, 864-873.

de Zubicaray, G. I., McMahon, K. L., Eastburn, M. M., \& Wilson, S. J. (2002). Orthographic/phonological facilitation of naming responses in the picture-word task: An event-related fMRI study using overt vocal responding. NeuroImage, 16, 1084-1093.

de Zubicaray, G. I., Wilson, S. J., McMahon, K. L., \& Muthiah, S. (2001). The semantic interference effect in the picture-word paradigm: An event-related fMRI study employing overt responses. Human Brain Mapping, 14, 218-227.

Duffau, H., Gatignol, P., Mandonnet, E., Peruzzi, P., TzourioMazoyer, N., \& CAPElle, L. (2005). New insights into the anatomofunctional connectivity of the semantic system: A study using corticosubcortical electrostimulations. Brain, 128, 797-810.

Eden, G. F., Joseph, J. E., Brown, H. E., Brown, C. P., \& Zeffiro, T. A. (1999). Utilizing hemodynamic delay and dispersion to detect fMRI signal change without auditory interference: The behavioral interleaved gradients technique. Magnetic Resonance in Medicine, 41, 13-20.

Elliott, M. R., Bowtell, R. W., \& Morris, P. G. (1999). The effect of scanner sound in visual, motor, and auditory functional MRI. Magnetic Resonance in Medicine, 41, 1230-1235.

Freire, L., Roche, A., \& Mangin, J. F. (2002). What is the best similarity measure for motion correction in $\mathrm{AMRI}$ time series? IEEE Transactions on Medical Imaging, 21, 470-484.

Friston, K. J., Glaser, D. E., Henson, R. N. A., Kiebel, S., PhilLIPS, C., \& Ashburner, J. (2002). Classical and Bayesian inference in neuroimaging: Applications. NeuroImage, 16, 484-512.

GANUSHCHAK, L. Y., \& SCHILlER, N. O. (2008). Brain error-monitoring activity is affected by semantic relatedness: An event-related brain potentials study. Journal of Cognitive Neuroscience, 20, 927-940.

Genovese, C. R., LaZar, N. A., \& Nichols, T. (2002). Thresholding of statistical maps in functional neuroimaging using the false discovery rate. NeuroImage, 15, 870-878.

GoLDRICK, M. (2006). Limited interaction in speech production: Chronometric, speech error, and neuropsychological evidence. Language \& Cognitive Processes, 21, 817-855.

GolDRICK, M. (2007). Connectionist principles in theories of speech production. In M. G. Gaskell (Ed.), The Oxford handbook of psycholinguistics (pp. 515-530). Oxford: Oxford University Press.

Hantsch, A., JescheniaK, J. D., \& Schriefers, H. J. (2005). Semantic competition between hierarchically related words during speech planning. Memory \& Cognition, 33, 984-1000.

Harley, T. A. (1993). Phonological activation of semantic competitors during lexical access in speech production. Language \& Cognitive Processes, 8, 291-309.

Harley, T. A., \& MACANDrew, S. B. G. (2001). Constraints upon word substitution speech errors. Journal of Psycholinguistic Research, 30, 395-418.

Harm, M. W., \& Seidenberg, M. S. (2004). Computing the meanings of words in reading: Cooperative division of labor between visual and phonological processes. Psychological Review, 111, 662-720.

Heim, S., Friederici, A. D., Schiller, N. O., Rüschemeyer, S. A., \& Amunts, K. (2009). The determiner congruency effect in language production investigated with functional MRI. Human Brain Mapping, 30, 928-940. doi:10.1002/hbm.20556

Hirschfeld, G. H. F., Jansma, B., Boelte, J., \& Zwitserlood, P. (2008). Interference and facilitation in overt speech production investigated with ERPs. NeuroReport, 19, 1227-1230.

InDEFREY, P., \& LEVELT, W. J. M. (2004). The spatial and temporal signatures of word production components. Cognition, 92, 101-144.

Jescheniak, J. D., Hantsch, A., \& Schriefers, H. J. (2005). Context effects on lexical choice and lexical activation. Journal of Experimental Psychology: Learning, Memory, \& Cognition, 31, 905-920.

Jescheniak, J. D., \& Schriefers, H. J. (1998). Serial versus cascaded processing in lexical access in language production: Further evidence from the coactivation of near-synonyms. Journal of Experimental Psychology: Learning, Memory, \& Cognition, 24, 1256-1274.

KAN, I. P., \& THOMPSON-SCHILL, S. L. (2004). Effect of name agreement on prefrontal activity during overt and covert picture naming. Cognitive, Affective, \& Behavioral Neuroscience, 4, 43-57.

Kuipers, J.-R., La HeIJ, W., \& Costa, A. (2006). A further look at semantic context effects in language production: The role of response congruency. Language \& Cognitive Processes, 21, 892-919.

Laird, A. R., McMillan, K. M., Lancaster, J. L., Kochunov, P., Turkeltaub, P. E., Pardo, J. V., \& Fox, P. T. (2005). A comparison of label-based review and ALE meta-analysis in the Stroop task. Human Brain Mapping, 25, 6-21.

Laurienti, P. J., Burdette, J. H., Wallace, M. T., Yen, Y. F., Field, A. S., \& Stein, B. E. (2002). Deactivation of sensory-specific cortex by cross-modal stimuli. Journal of Cognitive Neuroscience, 14, 420-429.

Levelt, W. J. M., Roelofs, A., \& Meyer, A. S. (1999). A theory of lexical access in speech production. Behavioral \& Brain Sciences, 22, 1-38.

Mahon, B. Z., Costa, A., Peterson, R., Vargas, K. A., \& CaraMAZZA, A. (2007). Lexical selection is not by competition: A reinterpretation of semantic interference and facilitation effects in the picture-word interference paradigm Journal of Experimental Psychology: Learning, Memory, \& Cognition, 33, 503-535.

McMahon, K., Pringle, A., Eastburn, M., \& Maillet, D. (2004). Improving EPI imaging quality and sound levels with bandwidth selection [Abstract]. In Proceedings of the 12th Annual Meeting of the International Society for Magnetic Resonance in Medicine, Kyoto, 1033. http://cds.ismrm.org/ismrm-2004.

Mechelli, A., Josephs, O., Lambon Ralph, M. A., McClelland, J. L., \& PrICE, C. J. (2007). Dissociating stimulus-driven semantic and phonological effects during reading and naming. Human Brain Mapping, 28, 205-217.

Meyer, A. S., \& Damian, M. F. (2007). Activation of distractor names in the picture-picture interference paradigm. Memory \& Cognition, 35, 494-503.

Meyer, A. S., \& Schriefers, H. (1991). Phonological facilitation in picture-word interference experiments: Effects of stimulus onset asynchrony and types of interfering stimuli. Journal of Experimental Psychology: Learning, Memory, \& Cognition, 17, 1146-1160.

Navarrete, E., \& Costa, A. (2005). Phonological activation of ignored pictures: Further evidence for a cascade model of lexical access. Journal of Memory \& Language, 53, 359-377.

Ojemann, J. G., Akbudak, E., Snyder, A. Z., McKinstry, R. C., RaIChle, M. E., \& Conturo, T. E. (1997). Anatomic localization and quantitative analysis of gradient refocused echo-planar fMRI susceptibility artifacts. NeuroImage, 6, 156-167.

Peterson, R. R., \& Savoy, P. (1998). Lexical selection and phonological encoding during language production: Evidence for cascaded processing. Journal of Experimental Psychology: Learning, Memory, \& Cognition, 24, 539-557.

RAPP, B., \& GOLDRICK, M. (2000). Discreteness and interactivity in spoken word production. Psychological Review, 107, 460-499.

Roelofs, A. (1992). A spreading-activation theory of lemma retrieval in speaking. Cognition, 42, 107-142.

RoElofs, A. (2003). Goal-referenced selection of verbal action: Model- 
ing attentional control in the Stroop task. Psychological Review, 110, 88-125.

Roelofs, A. (2008a). Attention to spoken word planning: Chronometric and neuroimaging evidence. Language \& Linguistics Compass, 2/3, 389-405. doi:10.111/j.1749-818x.2008.00060.x

Roelofs, A. (2008b). Tracing attention and the activation flow in spoken word planning using eye movements. Journal of Experimental Psychology: Learning, Memory, \& Cognition, 34, 353-368.

Rosinksi, R. R., Golinkoff, R. M., \& KUKISH, K. S. (1975). Automatic semantic processing in a picture-word interference task. Child Development, 46, 247-253.

Schriefers, H., Meyer, A. S., \& Levelt, W. J. M. (1990). Exploring the time course of lexical access in language production: Picture-word interference studies. Journal of Memory \& Language, 29, 86-102.

Shattuck, D. W., Mirza, M., Adisetiyo, V., Hojatkashani, C., SalaMON, G., NARR, K. L., ET AL. (2008). Construction of a 3D probabilistic atlas of human cortical structures. NeuroImage, 39, 1064-1080.

SNODGRASS, J. G., \& VANDERWART, M. (1980). A standardized set of 260 pictures: Norms for name agreement, image agreement, familiarity, and visual complexity. Journal of Experimental Psychology: Human Learning \& Memory, 6, 174-215.

Spalek, K., \& Thompson-Schill, S. L. (2008). Task-dependent semantic interference in language production: An fMRI study. Brain \& Language, 107, 220-228.

StaRreveld, P. A. (2000). On the interpretation of onsets of auditory context effects in word production. Journal of Memory \& Language, 42, 497-525.

Starreveld, P. A., \& La HeiJ, W. (1995). Semantic interference, orthographic facilitation, and their interaction in naming tasks. Journal of Experimental Psychology: Learning, Memory, \& Cognition, 21, 686-698.

Vaughan, J. T., Adriany, G., Garwood, M., Yacoub, E., Duong, T., DelaBarre, L., ET AL. (2002). Detunable transverse electromagnetic (TEM) volume coil for high-field NMR. Magnetic Resonance in Medicine, 47, 990-1000.

Vitkovitch, M., Rutter, M., \& Read, A. (2001). Inhibitory effects during object name retrieval: The effect of interval between prime and target on picture naming responses. British Journal of Psychology, 92, 483-506.

Weissman, D. H., Warner, L. M., \& WoldorfF, M. G. (2004). The neural mechanisms for minimizing cross-modal distraction. Journal of Neuroscience, 24, 10941-10949.

Zaitsev, M., Hennig, J., \& Speck, O. (2003). Automated online EPI distortion correction for fMRI applications [Abstract]. Proceedings of the 11th Annual Meeting of the International Society for Magnetic Resonance in Medicine, 1042. http://cds.ismrm.org/ismrm-2003.

\section{NOTES}

1. When we interrogated the separate peaks within this cluster, the pattern of BOLD signal responses across distractor conditions was identical to that overall (i.e., unrelated $>$ semantic $>$ phonological). Accordingly, we report responses here for the entire cluster.

2. The majority of PWI studies employ phonological distractors with initial consonant-vowel segments matching those of the target picture name. Thus, processing of the matching initial word-form information is facilitated in the phonological condition relative to the unrelated and semantically related conditions, in which the initial word-form mismatches that of the target picture name.

3. We did not observe activation of the pars orbitalis in either of our previous PWI experiments. It is possible that we were not able to image this region reliably with our older 2T MRI system because of EPI-related magnetic susceptibility artifacts (signal dropouts and geometric distortions; Ojemann et al., 1997).

4. This involves assuming that the semantically related distractor's lexical concept and those of its neighbors are inhibited as soon as they are accessed, because of lateral inhibition from the already selected target. If this were the case, one would also expect to observe reduced BOLD signal in the left MTG/STG for the semantically related versus unrelated comparison. Although this was observed numerically (see Figure 1), the difference was not statistically significant.

(Manuscript received January 25, 2009; revision accepted for publication March 16, 2009.) 